\title{
Abstracts for Southeast Pediatric Cardiology Society, Mission Inn Resort, Florida, 8-11 September 2004
}

\section{The incidence of chromosomal abnormalities in infants with congenital heart disease admitted to the cardiovascular Intensive Care Unit}

Jennifer Avari, ${ }^{1}$ Anthony Rossi, ${ }^{2}$ Parul Jayakar, ${ }^{3}$ Joanne Mora, ${ }^{4}$ Isabel De Leon, ${ }^{5}$ Elizabeth Welch ${ }^{2}$

Departments of ${ }^{1}$ Medical Education, ${ }^{2}$ Cardiology, ${ }^{3}$ Genetics, ${ }^{4}$ Cardiovascular Surgery and ${ }^{5}$ Cytogenetics, Miami Children's Hospital, Miami, Florida, United States of America

Background: Morbidity and mortality for patients with congenital heart disease is closely linked with chromosomal abnormalities. Recent advancements in cytogenetic techniques and the ability to determine mutations on specific loci have enhanced the care of infants with significant heart disease. This study was performed to determine the prevalence of chromosomal abnormalities in infants with complex congenital heart disease admitted to the Cardiac Intensive Care Unit (CICU) at Miami Children's Hospital. Methods: Chromosomal analysis and fluorescence in situ hybridization (FISH) studies were performed on 180 consecutive newborns admitted to the CICU (from January 2001 to August 2004). Chromosomes results and the presence of dysmorphic features were retrospectively reviewed. Patients were grouped according to their major cardiac lesion conforming with the classification used in the Baltimore-Washington Infant Study. Results: Patients were separated into 7 major groups: heterotaxy, outflow tract defects, atrio-ventricular canal (AVC), anomalous pulmonary venous return (APVR), right-sided obstructive defects, left-sided obstructive defects, and other. There were a total of 38 patients with chromosomal abnormalities $(38 / 180=21.1 \%)$; of these patients, $55 \%$ had deletion of chromosome 22q11.2, 29\% had Trisomy 21, and 16\% had other lesions (including Turners syndrome, Trisomy 18, Deletion 3, Deletion 11). All patients with chromosomal abnormalities were dysmorphic (p-value $<0.001$ ). We also had and additional 24 patients who were dysmorphic but for whom we were unable to document a chromosomal abnormality. Of the dysmorphic patients with normal chromosomes, many were diagnosed with syndromes, i.e. CHARGE, Noonan, and TAR. There was no statistical significance when classifying patients by race or gender. Conclusion: Though complex congenital heart disease is associated with chromosomal abnormalities, the need to screen all neonates with heart disease admitted to the CICU is not necessary. Chromosomal plus FISH analysis should be performed on patients with cardiac lesions that have a high yield for chromosome abnormalities, and when there are dysmorphic features on physical exam. Furthermore, Deletion 22q11.2 was the most common chromosomal abnormalities seen in neonates admitted to our CICU.

\section{Prenatal prediction of pulmonary hypoplasia with the maternal hyperoxygenation test}

R. Bader, J. C. Huhta

Department of Pediatrics, University of South Florida, St. Petersburg, Florida, United States of America

Background: Pulmonary hypoplasia (PH) is under development of lung tissue in the newborn. Maternal hyperoxygenation test (MHT) is used to assess fetal $\mathrm{PH}$ by having the pregnant women breath $100 \%$ oxygen and assessing the change in the blood velocities in the fetal pulmonary arteries after 30 weeks gestation. A $20 \%$ decrease 
in the pulsatility index (PI) of the PA in the fetus after 30 weeks gestation is normal. Hypothesis: Alveolar and arteriolar lung development occur together prenatally. Methods: MHT test was performed on 9 pregnant women (median maternal age 28.5 years) with fetuses (median gestation 31.5 weeks) with congenital anomalies that may cause PH (HLHLS $(n=2)$, LCDH $(n=2)$, Ebstein with pulmonary atresia $(n=1)$, TOF with absent pulmonary valve $(n=1)$, prune belly syndrome $(n=1)$, lung hypoplasia of unknown etiology $(n=2)$ ). The Doppler blood velocity pattern and PI in the first branch of the pulmonary artery were obtained before and 10-15 minutes after maternal breathing of $100 \%$ oxygen was obtained for each fetus at $\geqslant 30$ weeks GA. Results: Of the 9 fetuses tested, 5 (55\%) had a reactive MHT and all were delivered alive and well. Of the 4 fetuses (45\%) who had a nonreactive MHT 2 died in the early neonatal period and 1 had unknown outcome. Conclusion: Testing fetal pulmonary vascular reactivity with MHT is useful in assessing PH and in predicting neonatal outcome.

\title{
Early experience in the use of three-dimensional echocardiography in clinical characterization of left ventricular noncompaction
}

\author{
G. H. Baker, N. Pereira, A. M. Hlavacek, K. Chessa, G. Shirali \\ Children's Hospital, Medical University of South Carolina, Charleston, South Carolina, United States of America
}

Background: Numerous modalities have been used to diagnose and characterize left ventricular noncompaction (LVNC) including MRI, two-dimensional echocardiography (2DE), contrast-enhanced 2DE, and angiography. A variety of diagnostic criteria have been proposed for $2 \mathrm{DE}$. The current case series examines the use of threedimensional echocardiography (3DE) in four such cases of LVNC. Methods: From December 2003 to March 2004, we performed real-time 3DE using a Phillips Sonos 7500 echocardiographic scanner equipped with a 2-4 MHz 3D matrix array, transthoracic probe, to evaluate four patients with LVNC. The real-time 3D transthoracic probe allows for data set acquisition in an ultrasound wedge approximately $46^{\circ}$ by $23^{\circ}$. In addition, complete $3 \mathrm{D}$ volume rendering is acquired allowing for instantaneous volumetric imaging. Results: The patients' ages ranged from 2 months to 42 years, with body surface areas ranging from $0.28 \mathrm{~m}^{2}$ to $1.66 \mathrm{~m}^{2}$. One patient was found to have further involvement of the right ventricle. Another patient had coarctation of the aorta and bicuspid aortic valve. In all four patients, real-time 3D transthoracic echocardiography enabled diagnosis as well as providing detailed characterization of the affected myocardium. Entire trabecular projections and intratrabecular recesses were easily visualized simultaneously and endocardial borders were clearly demarcated. Wall motion abnormalities of affected myocardium were clearly visualized. The compacted and noncompacted portion of the myocardium could be differentiated well. Conclusion: Our study provides preliminary data highlighting the utility and feasibility of real-time 3D transthoracic echocardiography in the clinical characterization of LVNC. The complex 3D nature of this disorder, and the endocardial hypertrabeculation were readily visualized.

\section{Pre- and post-operative quantitation of right ventricular tissue Doppler velocities in infants with obstructive left heart lesions}

D. Christensen, ${ }^{1}$ B. Cardis, ${ }^{1}$ W. Mahle, ${ }^{1}$ R. Lewis, ${ }^{2}$ J. Huckaby, ${ }^{2}$ D. Fyfe ${ }^{1}$

${ }^{1}$ Children's Healthcare of AtlantalEmory University and ${ }^{2}$ Children's Healthcare of Atlanta, Atlanta, Georgia, United States of America

Background: The purpose of this study was to use tissue Doppler imaging (TDI) to serially quantitate initial and pre-op right ventricular (RV) TDI velocities and compare them to post-op RV TDI velocities as measures of RV function in newborns with obstructive left-sided cardiac defects. Methods: Fifteen patients were prospectively studied with diagnoses of hypoplastic left heart $(n=12)$ and arch interruption (2) or severe coarctation (1). TDI systolic (S) and early diastolic (E) velocities were recorded at the tricuspid annulus and the ventricular 
septum at: (1) admission, (2) immediately pre-op and (3) during recovery. Velocities were compared using repeated measure ANOVA tests. Results: Mean age at diagnosis was 1 day (0-4 days). Time from diagnosis to surgery was 4.8 days (2-12 days), age at surgery 5.4 days (2-13 days), and time from surgery to post-op echo 12.3 days (3-19 days). Data are shown in table. Tricuspid annular $\mathrm{S}$ velocities increased from admission to preop, but tricuspid annular E velocites did not change significantly. Both $\mathrm{S}$ and $\mathrm{E}$ velocities decreased postoperatively. No significant changes occurred in the ventricular septal systolic (S) or diastolic (E) velocities from admission to pre-op, but septal (S) velocities declined post-operatively. Conclusion: These data quantitate that following the institution of PGE and initial medical and ventilatory management, increase is seen in RV annular systolic and diastolic velocities, and that these velocities decline significantly post-operatively. Initial septal systolic and diastolic velocities were severely abnormal and did not significantly change pre and post-operatively. These data may have significance for both post-op and subsequent long term RV function.

\begin{tabular}{|c|c|c|c|c|}
\hline & Initial & Pre-op & Post-op & p-value \\
\hline $\mathrm{TV}(\mathrm{S}) \mathrm{cm} / \mathrm{s}$ & $5.8(.50)$ & $7.9^{*}(.50)$ & $4.7^{* *}(.27)$ & $\begin{aligned} * & =0.003 \text { vs. initial }(0.62) \\
* * & =<0.0001 \text { vs. pre-op }(0.55)\end{aligned}$ \\
\hline $\mathrm{TV}(\mathrm{E}) \mathrm{cm} / \mathrm{s}$ & $-10.9(.74)$ & $-12.8^{*}(.99)$ & $-7.5^{* *}(.63)$ & $\begin{aligned} * & =0.09 \text { vs. initial }(1.1) \\
* * & =0.00003 \text { vs. pre-op }(1.2)\end{aligned}$ \\
\hline Septum $(\mathrm{S}) \mathrm{cm} / \mathrm{s}$ & $4.3(1.1)$ & $4.7^{*}(1.1)$ & $3.7^{* *}(1.1)$ & $\begin{array}{l}{ }^{*} \text { NS vs. initial }(1.1) \\
{ }^{* *}=0.009 \text { vs. pre-op }(1.1)\end{array}$ \\
\hline Septum (E) & $-8.1(1.1)$ & $-7.6(1.1)$ & $-7.8(1.1)$ & NS \\
\hline
\end{tabular}

()$=$ standard error

\section{Outcomes following electroanatomic mapping and ablation for the treatment of ectopic atrial tachycardia in the pediatric population}

Rose M. Cummings, Melinda Dobbs, Margaret J. Strieper, Robert M. Campbell, Lynne Costello, Virginia Balfour, Amanda Burchfield, Patricio A. Frias

\section{Sibley Heart Center, Children's Healthcare of Atlanta, Emory University, Atlanta, Georgia, United States of America}

Background: Ectopic atrial tachycardia (EAT) is often resistant to medical therapy, with radiofrequency ablation (RFA) being a preferred treatment option. Three-dimensional electroanatomic (3-D) mapping was introduced as a tool for improved substrate localization. To date, there are no published data reporting this technology in pediatric pts with EAT. The objective of this study was to examine our experience with 3-D mapping and standard mapping in this population. Methods: Retrospective review of pediatric pts with EAT requiring RFA from 1993 to present. We analyzed method of ablation, acute success and recurrence rates, procedure and fluoroscopy times, and cardiac function. Results: Twenty-five pts underwent 31 RFA procedures. All pts have been followed for $>6$ mos $(6$ mos7 yrs). Standard mapping (Group 1) was used in 11 pts (5F/6M, 1.4-11.8 yrs) who underwent 13 RFA procedures; 3-D mapping (Group 2, Oct 2000-present) was used in 16 pts (8F/8M, 2.7-17 yrs) who underwent 18 RFA procedures. Left-sided focus was present in 6/13 in Group 1 and 7/18 in Group 2 (all transeptal, NS). There was a trend toward fewer lesions ( $15 \pm 14$, median 9.5 in Group 1; $8 \pm 6$, median 6.5 in Group 2) with 3-D mapping (NS). There were no differences in acute success rates (10/13 Group 1 vs. 18/18 Group 2, NS). However, recurrence or persistence of tachycardia at intermediate follow-up ( $2 \mathrm{wk}-1 \mathrm{yr}$ ) was documented in 7 of 13 cases in Group 1, compared to only 2 of 18 cases in Group $2(\mathrm{p}=0.01)$. Six pts underwent repeat RFA: 2 pts using standard mapping (1 failure, 1 success), and 4 pts using 3-D mapping [all acute and long-term ( $>1$ yr) success]. Procedure (232 \pm 84 vs. $268 \pm 72 \mathrm{~min}$, skin-to-skin) and fluoroscopy ( $47 \pm 24 \mathrm{vs.} 40 \pm 20 \mathrm{~min})$ times were similar (NS). Of the 25 pts, 17 (7 - Group 1, 10 - Group 2, NS) presented with cardiomyopathy (EF 38.6 $\pm 12.1 \%$ ). Successful RFA resulted in improved EF $(61.1 \pm 11.6 \%, \mathrm{p}<0.0001)$ in the $14 \mathrm{pts}$ in whom pre and post RFA echos were available. Conclusion: Compared to standard techniques, 3-D electroanatomic mapping has resulted in no acute failures, statistically reduced recurrence rates and improved overall success in the management of EAT. 


\title{
A new Eb curved balloon to curve stents across the aortic arch
}

\author{
M. R. Ebeid, K. Adcock, R. Berry, C. Gaymes, J. Joransen \\ University of Mississippi Medical Center, Jackson, United States of America
}

Introduction: During stenting of coarctation, not infrequently the stent has to be placed across the curved segment of the aortic arch. This may result in stent protrusion, embolization or unnecessary arch straightening. It would be beneficial if the stent would follow the natural curve of the arch. Curving the stent using current balloons is not possible. The study was designed to test the new Eb curved balloon to assist in stent curving. Methods: Four swine animals were included in the study. Four stents were implanted along the aortic arch curvature (1 EV3 Max stents $^{\circledR}, 1 \mathrm{EV} 3 \mathrm{Mega}^{\circledR}, 1$ Palmaz $3110^{\circledR}$ and 1 Genesis $\mathrm{XD}^{\circledR}$ ). Three stents were implanted using the regular straight balloon and subsequently curved using the Eb curved balloon. One stent was placed primarily using the $\mathrm{Eb}$ curved balloon. One pig died during the procedure before using the Eb curved balloon. The procedure was continued. On necropsy there was no rupture or obvious complications. Cause of death is unknown. Results: In all stents, the Eb curved balloon oriented itself appropriately across the curve of the arch following the wire curve; with the convex side oriented cephalic. On fluoroscopy, the curvature of the EV3 stents $\left(\mathrm{Max}^{\circledR}, \mathrm{Mega}^{\circledR}\right)$ and the Genesis $\mathrm{XD}^{\circledR}$ was more apparent and maintained after deflating the balloon. The Palmaz 3110 seemed more resilient to maintaining the curve. On necropsy the stents were in the appropriate places with no rupture (including the pig which died). The curvature of the stent was more apparent in the Max ${ }^{\circledR}, \mathrm{Mega}^{\circledR}$ and the Genesis $\mathrm{XD}^{\circledR}$ stents. The Palmaz ${ }^{\circledR}$ stent was less curved. Conclusion: The use of Eb curved balloons to curve the stent appropriately across the aortic arch curvature is feasible. In the animals we tested, the balloon curve followed the wire curvature. The Palmaz 3110 may be more resilient than the EV3 stents or the Genesis $\mathrm{XD}^{\circledR}$. Curving of the stent across the arch may decrease potential complications. Further studies are needed.

\section{Implementation of the DeBakey pediatric left ventricular assist device}

D. M. Edwards, E. S. Hale

Cardiac Surgery Specialty Nurse, Arkansas Children's Hospital, Little Rock, Arkansas, United States of America

Purpose: To provide nursing staff the knowledge and skills required to care for a pediatric patient pre and post left ventricular assist device (LVAD) placement as well as collect required data for MicroMed to monitor and document patient impact/response as required by the FDA before commercialization is granted. Description: Arkansas Children's Hospital is implementing the use of the DeBakey VAD child, which is currently in clinical trials, to help patients with end stage heart failure. Eligibility criteria include but are not limited to, body surface area $(B S A) \geq 0.7$, age $5-16$, and device to be used as a bridge to transplant for patients who present with end stage heart failure. Due to the additional skills and equipment necessary to care for these patients, staff was educated with frequent review and evaluation of things such as the use and care of thermodilution catheters, the DeBakey VAD child equipment, systemic venous oxygenation $\left(\mathrm{SVO}_{2}\right)$ monitoring, as well as familiarity with the equipment and data collection tools. Thermodilution catheters will be used to collect required and pertinent data before and after device implantation such as cardiac index, pulmonary artery capillary wedge pressure, systemic vascular resistance index, and pulmonary vascular resistance index. $\mathrm{SVO}_{2}$ monitoring provides valuable information regarding oxygen supply/consumption. Policy and procedures as well as patient plans of care were also written to satisfy institutional guidelines. Summary: Although Arkansas Children's Hospital Heart Center has not yet implanted the DeBakey LVAD, there continues to be ongoing education and initiation of policies/procedures. The final presentation will include implementation should the opportunity arise. Arkansas Children's Hospital Heart Center projects to have 1-3 DeBakey VAD child devices implanted per year. Conclusion: Arkansas Children's Hospital Heart Center is participating in the data collection for the DeBakey VAD child in conjunction with other pediatric sites in the United States, until there are 50 patients nation wide who participate. At that time Arkansas Children's Hospital Heart Center will continue to provide this service as well as utilize other assist devices to 
provide cutting-edge care to our patients. These assist devices may potentiate/replace extracorporeal membrane oxygenation (ECMO) as a bridge to transplant in some of our patient population.

\title{
Transcatheter closure of mid-muscular ventricular septal defects with the Amplatzer atrial septal defect occluder
}

\author{
J. A. Ettedgui, E. M. Greeley \\ University of FloridalJacksonville, Wolfson Children's Hospital, Jacksonville, Florida, United States of America
}

Background: The Amplatzer ASD occluder was approved by the Food and Drug Administration for transcatheter closure of ASD in 2001. The device for closure of muscular VSD is still in clinical trials. Purpose: Use of Amplatzer ASD occluder for closure of mid-muscular VSD in children. Materials and methods: Transcatheter closure of muscular VSD was attempted in 2 children. Patient 1 was a 3-month-old infant with multiple muscular trabecular VSDs and congestive heart failure. Qp/Qs was $>4: 1$. Patient 2 was a 5-year-old girl with a restrictive single mid-trabecular VSD with volume overload to the left ventricle and a Qp/Qs of 2:1. Catheterization was performed via the femoral vessels and the right internal jugular vein under general anesthesia. Transesophageal echocardiography was used in both patients. The VSD was crossed from the left ventricular side, a guide wire snared in the right ventricle and exteriorized via the internal jugular vein. A long sheath was advanced through the internal jugular vein over the wire into the left ventricle. The procedure was successful in both. A 9-mm device was used in patient 1 and a 7-mm device in patient 2. Results: Patient 1 has been followed for 18 months. Heart failure was controlled and there is minimal residual shunting through small additional muscular VSDs. Patient 2 has been followed for 15 months and there is no residual shunting. There is no evidence of wire fracture on chest X-ray. Conclusions: In selected patients the Amplatzer ASD device may be a valid alternative for transcatheter closure of muscular VSD.

\section{Atrial tachyarrhythmias during transcatheter closure of atrial septal defects with the Amplatzer device}

\author{
J. A. Ettedgui, E. M. Greeley \\ University of FloridalJacksonville, Wolfson Children's Hospital, Jacksonville, Florida, United States of America
}

Background: Atrial tachyarrhythmias are one of the long-term complications of an untreated ASD. They can also occur during catheter manipulation within the atria. Purpose: Establish the incidence of atrial tachyarrhythmias during transcatheter closure of ASD with the Amplatzer Septal Occluder and note differences between children and adults. Materials and methods: Transcatheter closure of ASD using the Amplatzer device was performed in 59 patients from June 2002 to June 2004. There were 45 patients under 21 years of age (mean $=7$ years, range $=8$ months-21 years) and 14 were over 21 (mean $=47$ years, range $=22-68$ years). Results: There was no difference in $\mathrm{Qp} / \mathrm{Qs}$ between both groups $(1.88 \pm 0.81$ vs $1.92 \pm 1.04, \mathrm{p}=0.88)$ or in mean pulmonary artery pressure $(17.43 \pm 6.3$ vs $15.0 \pm 4.3, \mathrm{p}=0.16)$. Five patients $(8.5 \%)$ had atrial tachyarrhythmias during device closure; $2 / 45$ under 21 years and $3 / 14$ over 21 years $\left(\chi^{2}=3.1, p=0.07\right)$. Cardioversion was required in 3 patients ( 1 under 21 and 2 over 21) and sinus rhythm resumed spontaneously in the other 2 ( 1 in each group). None of the patients with atrial tachyarrhythmias during device closure have had recurrent tachycardia during the follow up period. Conclusions: Atrial tachyarrhythmias are a common complication during transcatheter closure of ASD using the Amplatzer device. There is a trend towards a higher incidence in adults undergoing this procedure. A larger study group may be required to reveal a significant difference. Atrial tachyarrhythmias during device closure do not predict future development of tachycardia. 


\title{
Resynchronization and strain recovery following reimplantation of anomalous coronary artery
}

B. K. Fornwalt, W. T. Mahle, D. A. Fyfe

Emory University School of Medicine, Children's Healthcare of Atlanta and Sibley Heart Center, Atlanta, Georgia, United States of America

Purpose: To characterize abnormalities of global left ventricular (LV) synchrony and segmental contractility in a patient with anomalous left coronary from the pulmonary (ALCAPA) before and after reimplantation using real-time 3D echo (3DE) and Tissue Doppler Velocity imaging (TVI). Methods: 3DE and TVI data from the LV were collected on admission from a 6-week-old patient with ALCAPA and 13 and 35 days after reimplantation. Regional volume curves of the 16 standard LV segments were generated. TVI data was collected from apical 2, 3 and 4-chamber views. Systolic function was assessed with mid-wall peak strain, and diastolic function was assessed using peak early diastolic strain rate. The 12 non-apical segments were grouped according to the 3 coronary artery territories for strain analysis. Global LV synchrony was characterized by the standard deviation (SD) of times to peak systolic tissue velocity in the 12 non-apical segments and the SD of times to peak ejection fraction (EF) from the 3D segments. Results: Global LVEF improved from 13\% pre-op to 54\% five weeks after reimplantation. The right coronary artery (RCA) EF increased from 28 to $53 \%$, the left circumflex (LCX) from 13 to $62 \%$, and the left anterior descending (LAD) from 15 to $52 \%$. The SD of times to peak EF declined from 104 to $34 \mathrm{~ms}$ and SD of times to peak systolic tissue velocity declined from 51 to $14 \mathrm{~ms}$ (Fig. 1). Derivatives of the regional volume curves validated resynchronization. Conclusions: The initial severe LV dyssynchrony in a patient with anomalous coronary progressively resolved following coronary reimplantation and paralleled myocardial functional recovery in specific coronary territories (Fig. 2). Tissue Doppler and real-time 3D echocardiographic methods to quantify global intra-ventricular synchrony were in close agreement.

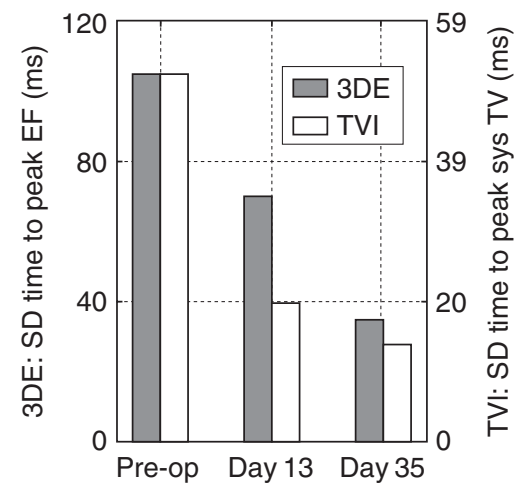

Figure 1.

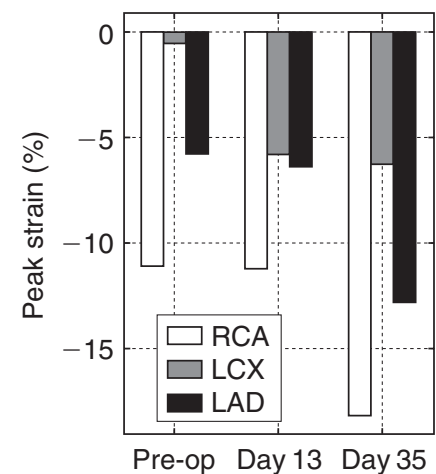

Figure 2 .

\section{Outcome comparison of antibiotic impregnated central venous line with non- impregnated central venous line in the pediatric Cardiac Intensive Care Unit}

\author{
A. Cuadrado, ${ }^{1,2}$ J. Huckaby ${ }^{2}$ \\ ${ }^{1}$ Emory University School of Medicine and ${ }^{2}$ Children's Healthcare of Atlanta, Atlanta, Georgia, United States of America
}

After adopting the practice of inserting antibiotic impregnated central venous lines (CVL) in the Cardiac Intensive Care Unit (CICU) at Children's Healthcare of Atlanta, a report from the Infection Control Department revealed that there was a significant decrease in blood stream infections. The purpose of this study was to determine if the use of this CVL also reduced the following: (1) days antibiotics received; (2) days mechanical ventilation required; (3) days inotropic support required; or (4) days in the CICU. Methods: A retrospective chart review was conducted. The patients were divided into two groups. The treatment group was defined as being in the CICU and an anti-biotic impregnated CVL placed. The control group was defined as 
being in the CICU prior to the use of this catheter and had the non-impregnated catheter placed. Of the 78 charts reviewed, 38 patients were in the treatment group, 40 in the control.

Results:

\begin{tabular}{|c|c|c|}
\hline & Treatment (38) & Control (40) \\
\hline Average length of stay: & 29.5 (median 24) & $23.5($ median 17$)$ \\
\hline At least 1 blood stream infection (BSI) & 11 & 13 \\
\hline - Required reintubation \pm 24 hours from initial BSI & $2(18 \%)$ & $1(8 \%)$ \\
\hline - Antibiotics added or changed \pm 24 hours from initial BSI & $9(82 \%)$ & $10(77 \%)$ \\
\hline - Inotropes added or changed \pm 24 hours from initial BSI & $3(27 \%)$ & $2(15 \%)$ \\
\hline
\end{tabular}

Conclusion: Although the number of blood stream infections did decrease overall, it did not reduce antibiotic use, inotropic use, ventilator days or CICU stay. Limitations of this study were the fact that it was a retrospective chart review and only a select group was studied. The Infection Control Department looked at the whole CICU population. Further evaluation is warranted.

\title{
Use of the Safeguard ${ }^{\mathrm{TM}}$ pressure assisted dressing in the pediatric cardiac catheterization lab
}

L. Lam, D. Nykanen, A. Hernandez, N. Ventrua, R. Whalen, E. Zahn

Miami Children's Hospital, Miami, Florida, United States of America

Purpose: The purpose of this study is to review our experience with the use of Safeguard ${ }^{\mathrm{TM}}$ post pediatric cardiac catheterization and evaluate it's effectiveness for achieving hemostasis. Method: A retrospective review including cath database reconciled with meticulous tracking of clinical events including data from hospital charts, performance improvement reports with quarterly documentation of variances and complications following catheterization were utilized. Results: From 03/17/2004 to 08/06/2004 85 patients undergoing 96 procedures had a Safeguard ${ }^{\mathrm{TM}}$ applied to 124 femoral sites to maintain hemostasis following cardiac catheterizations. At time of catheterization patient mean age was $10.4 \pm 10.7$ years (range 3 months to 46 years) and mean weight was $34.8 \pm 23.9 \mathrm{~kg}$ (range 6.0 to $122.3 \mathrm{~kg}$ ). Access site of Safeguard ${ }^{\mathrm{TM}}$ application was venous only $(\mathrm{n}=63)$, arterial only $(\mathrm{n}=20)$ or both artery and vein $(\mathrm{n}=41)$. Sheath size ranged from $4 \mathrm{Fr}$ to $11 \mathrm{Fr}$. Rebleeding after leaving the cardiac catheterization lab was rare $(n=1)$. No complications related to the Safeguard ${ }^{\mathrm{TM}}$ were encountered. Based on this experience we reviewed our incidence of rebleeding in the quarter immediately before our use of the Safeguard ${ }^{\mathrm{TM}}$ device. In that quarter we experienced 7 rebleeds, 1 patient in October, 3 patients in November, and 3 patients in December. All of which would have received a Safeguard ${ }^{\mathrm{TM}}$ device in the current era. Perceived advantages to Safeguard ${ }^{\mathrm{TM}}$ use from the nursing staff were maintenance of hemostasis with the active and pediatric patient, ability to visualize access site, maximizing valuable staff resources, allows increased mobility of patient, easy application and maintenances with simple adjustment of pressure, decreases length of stay, decreased hospital costs, decreased patient and parent fear from occurrences of rebleeds. Conclusion: We believe Safeguard ${ }^{\mathrm{TM}}$ is a safe and an effective mechanism to achieve hemostasis in the pediatric patient. It has become the standard of care in all of our patients.

\section{Cardiovascular profile score predicts myocardial dysfunction in fetuses with congenital heart disease}

\author{
R. Martinez, E. Kales, J. C. Huhta \\ University of South Florida, Department of Pediatrics, St. Petersburg, Florida, United States of America
}

Background: A ten-point score in five categories (heart size, venous Doppler, hydrops, cardiac function, and arterial Doppler) similar to the APGAR score can measure the severity of fetal congestive heart failure and aid in prenatal prognosis assessment. Congenital heart disease (CHD) is associated with progressive heart failure in utero in about $5 \%$ of patients. Objective: Look for prehydropic signs of myocardial dysfunction in fetuses with 
CHD and evaluate the CVP. Methods: CVP was scored on 45 nonhydropic fetuses with CHD, left heart -9 , right heat - 15, TGA - 4, others - 17 at 65 different occasions between the time of diagnosis of the defect and delivery (gestational age 18-38 weeks). Each had assessment of the myocardial performance index (MPI) in both right $(\mathrm{RV})$ and left $(\mathrm{LV})$ ventricles. MPI = ratio of the isovolumic time to the ejection time. Normal MPI does not change with gestational age. Results: MPI ranged from 0.27 to 0.93 (normal $<0.40$ ) and CVP from 6 to 10. There was an inverse correlation between the RV CVP and MPI $(r=0.54)$ with a lower CVP score associated with a higher MPI suggesting systolic and/or diastolic dysfunction. There was no correlation with LV MPI. Conclusion: A decreasing CVP score is associated with signs of fetal RV myocardial dysfunction. Improved fetal cardiac function could occur with transplacental medical therapy.

\title{
Cigarette smoke as a risk factor for coronary artery disease and sudden unexpected death in fetuses and infants
}

\author{
L. Matturri, G. Ottaviani, R. Mingrone, M. Mauri, A. M. Lavezzi \\ Institute of Pathology, University of Milan, Italy
}

Maternal smoking during pregnancy makes it more likely that the baby will be stillborn or die in the first year of life. Cigarette smoke is the most important risk factor for fetal and infant sudden death (SIDS). The risk of stillbirth or death during the infant's first year of life is directly related to the amount the mother smoked. The present study adds significant evidence on the need to avoid cigarette smoking. The pathogenic mechanism of smoke is referable to different factors. The combustion products of nicotine, in addition to their diffuse toxicity, are heterogeneous and cause specific lesions of the autonomic nervous system. Gaseous combustion products, such as carbon oxide, lead to atherosclerotic plaques in the cardiovascular district and in the sino-atrial and atrio-ventricular arteries. Consequently, these combustion products cause an oxygenation deficit of the common myocardium, as well as developmental abnormalities of the conducting tissue, laying the morphological substrate for arrhythmias. Our study population included 22 stillborns and 49 infants dying suddenly and unexpectedly. All cases died since cause between the 32nd week of gestation and one year of age. Samples of the myocardium and the major coronary arteries (left main, left anterior descending, left circumflex, right main, right posterior descending, right marginal) were stained with hematoxylin-eosin and trichromic heidenhain (Azan). The cardiac conduction system was removed in two blocks: the first included the sino-atrial node and the crista terminalis, the second contained the atrioventricular node, His bundle down to the bifurcation and bundle branches. These two blocks were cut serially at intervals of 40- $\mu \mathrm{m}$ (levels) and stained alternately with hematoxylin-eosin and Azan. In $55 \%$ of fetuses and in $67 \%$ of the infants, multifocal coronary early atherosclerotic lesions of varying entity were detected. The alterations ranged from focal plaques with mild myointimal thickening to juvenile soft plaques in infants reducing the arterial lumen. In $45 \%$ of stillborns and in $75 \%$ of infants with coronary lesions the parents were smokers. A significant correlation was observed between early atherosclerotic lesions and cigarette smoking. The reduction in the coronary lumen can be such as to cause alterations in cardiac blood supply. These early atherosclerotic lesions can be attributed to a direct action of the combustion products of nicotine on the smooth muscle cells of the tunica media of the arterial walls and/or on the neurons, interfering with homeostasis and cell differentiation, as well as to an indirect action of hypoxemia induced by arterio- and arteriolosclerosis. The harmful effects of cigarette smoking are not confined to the coronaries but also affect the small and medium-caliber arteries, including the sino-atrial and atrio-ventricular arteries. Analysis of our series suggests that parental cigarette smoking has the highest significance among the risk factors considered in the pathogenesis of sudden fetal and infant death, while the newborn's position in the crib, which has been assigned a fundamental importance in recent years, is not equally supported by anatomo-pathologic data.

\section{Pulmonary artery size and Fontan completion: a time for reevaluation}

\author{
S. Maurer, ${ }^{1}$ V. Vasuki, ${ }^{1}$ V. Bandisode,${ }^{1}$ A. Atz,${ }^{1}$ S. Bradley, ${ }^{1}$ M. Stroud, ${ }^{1}$ S. Colan, ${ }^{2}$ G. Shirali ${ }^{1}$ \\ ${ }^{1}$ Medical University of South Carolina, Charleston, South Carolina; ${ }^{2}$ Children's Hospital Boston, Boston, \\ Massachusetts, United States of America
}

There is limited contemporary data on the relevance of pulmonary artery (PA) size for patients with functionally single ventricle (SV) who are being staged towards Fontan palliation. We measured indexed PA sizes by 
angiography (Nakata index (NI)) in the course of staged Fontan palliation among a contemporary cohort of patients with SV, and compared their NI to previously published echocardiographic (echo) and angiographic (cath) normal values. Methods: We reviewed charts on all patients who underwent cavopulmonary anastomosis $(\mathrm{CPA})(\mathrm{n}=106)$ or Fontan completion $(\mathrm{n}=86)$ from $6 / 96$ to 5/04. PA diameters in every patient were measured at cath prior to CPA and Fontan and their NI values were calculated. NI from cath prior to both operations were then compared to previously published echo measurements of PA diameters from a group of 256 children without heart disease. The latter diameters were indexed to body surface area (BSA), thus yielding the echo equivalent of the NI. Separately, NI from study patients were compared to values previously reported by Nakata. Results: Median weight at CPA was $6.1(4.0-16.9) \mathrm{kg}$ and at Fontan was 13.0 (6.25-23.4) kg. Median BSA at CPA was $0.33(0.23-0.61) \mathrm{m}^{2}$ and at Fontan was $0.56(0.33-0.89) \mathrm{m}^{2}$. At pre-CPA cath, the mean NI was $192.2 \pm 88 \mathrm{~mm}^{2} / \mathrm{m}^{2}$, which was not significantly different from the NI obtained by echo in children with normal hearts $\left(175 \pm 54 \mathrm{~mm}^{2} / \mathrm{m}^{2}, \mathrm{p}=0.125\right)$. At pre-Fontan cath, the mean NI was significantly greater than the NI obtained by echo in normals $\left(211.1 \pm 78 \mathrm{~mm}^{2} / \mathrm{m}^{2}\right.$ vs. $\left.178 \pm 50 \mathrm{~mm}^{2} / \mathrm{m}^{2}, \mathrm{p}<0.001\right)$. Nakata et al. previously reported a normal NI of $330 \pm 30 \mathrm{~mm}^{2} / \mathrm{m}^{2}$ and concluded that NI $\geqslant 250 \mathrm{~mm} / \mathrm{m}^{2}$ was needed for acceptable Fontan results. In our group, hospital survival following Fontan was 97\% (84 of 87) despite only $28 \%$ (24 of 87 ) patients meeting the historic NI threshold. Conclusions: Cath-derived PA sizes pre-CPA are similar to echo-derived normal PA sizes, and exhibit inter-stage growth to above-normal size pre-Fontan. The historically accepted NI does not reflect observed PA sizes in a contemporary cohort undergoing staged Fontan palliation. The existence of a threshold NI is unknown but probably deserves re-definition in the current era.

\section{Echocardiographic diagnosis, natural history and prognosis of fetuses with single ventricle}

P. L. Ferrer, ${ }^{1,2}$ A. Parthiban, ${ }^{1}$ S. Sinisterra, ${ }^{1}$ J. Osit, ${ }^{2}$ O. Garcia ${ }^{1,3}$

Departments of ${ }^{1}$ Pediatrics, ${ }^{2}$ Obstetrics and ${ }^{3}$ Pathology, University of Miami School of Medicine, Miami, Florida, United States of America

Objectives: To evaluate the frequency, presentation, natural history and prognosis in fetuses with single ventricle (SV). Methods: A retrospective review of 3,607 echocardiograms in 2,215 fetuses (1983-2004) found 483 fetuses with congenital heart disease (CHD). Twenty fetuses with SV (atrioventicular univentricular connection) were selected for presentation. Detailed quantitative information including cardiac area, cardiothoracic ratio, chamber size, wall thickness, ventricular function parameters, color flow imaging, and Doppler velocities in the foramen ovale, ventricular inflows, great arteries, and ductus were compared with a control group of 91 normal fetuses according to gestational age (GA). Diagnosis was confirmed by neonatal echocardiogram and in some cases by angiography, surgery and/or autopsy. Results: The diagnosis of SV was made at a median GA of 26.7 weeks (range 19-38.5 weeks). Levocardia was present in 17 fetuses and dextrocardia in three. (A) Seven fetuses had single left ventricle all with situs solitus of the atria; five fetuses had two patent atrioventicular valves (AVV) and two had one valve patent and the other imperforated. In one case with dextrocardia and L-ventricular loop (ventricular inversion), there was hypoplasia of the tricuspid valve; four fetuses had D-malpositon of the great arteries (D-MGA) and one fetus L-MGA concordant with the position of the rudimentary right ventricular outflow. Three fetuses had pulmonary atresia and three fetuses had aortic hypoplasia (one with aortic atresia). (B) Eleven fetuses had single right ventricle all with situs solitus of the atria. Two fetuses had two patent $\mathrm{AV}$ valves and nine fetuses had one valve patent and the other imperforated. Four fetuses had a hypoplastic left ventricle with absence of inflow or outflow; the remaining seven fetuses had no demonstrable left ventricle. There was right ventricular enlargement and hypertrophy; both great arteries were connected with the right ventricle. The great arteries were normally related in six fetuses and malposed in five. One fetus had pulmonary atresia, and one had pulmonic stenosis; four had a hypoplastic aorta (3 with aortic atresia), two had interruption of the aortic arch and one had coarctation. Three fetuses had heterotaxia and four had anomalous connection of the pulmonary veins (APVR). (C) Two fetuses had common ventricle; one with levocardia, right AVV atresia and D-MGA; the other had dextrocardia, common AVV and "primitive ventricle" associated with L-MGA, complete AV block and polysplenia. Of the 20 fetuses, six had fetal demise (2 stillbirth, 4 termination), in four the outcome is uncertain, two were neonatal deaths prior to surgery and 11 underwent palliative surgery (Norwood with/without Damus-Kaye-Stansel procedure, shunts, correction of APVR, Glenn and Fontan procedures). Four infants survived, four died in the postoperative period and three were lost 
to follow-up. Conclusions: Prenatal diagnosis of single ventricle (atrioventicular univentricular connection) is infrequent ( $0.99 \%$ of referrals, $4.6 \%$ of fetuses with CHD). Diagnosis is challenging. The morphology and function may evolve with time (acquired pulmonary atresia). SV requires a variety of surgical approaches. In spite of progress, surgical therapy is only palliative. Overall prognosis is still somber with a survival rate of $20 \%$ in patients with known follow-up.

\title{
ECMO orientation for pediactric nurses
}

\author{
C. A. Pye
}

\section{Arkansas Children's Hospital, Little Rock, Arkansas, United States of America}

Arkansas Children's Hospital (ACH) performed over 60 extracorporeal membrane oxygenation (ECMO) cases for the year of 2003. The ECMO unit is a free standing clinical area designated to the care of patient's requiring ECMO support for survival. The patient population comprising of the 60 cases were $65 \%$ cardiac and $35 \%$ respiratory in nature. The patients were from the different units of the Cardiovascular Intensive Care Unit, Pediatric Intensive Care Unit, and the Neonatal Intensive Care Unit. Due to the nature of the different disease processes and patient ages, the nursing staff for these unique patients originates from the unit of patient origin. An assessment of the educational needs from the nurses who worked in the ECMO unit revealed the need for a formal orientation bedside reference manual. A formal orientation bedside reference manual consisting of new and revised polices was developed with the individual needs of each unit and patient population as a focus. Multiple, individual unit inservices were provided to the nursing staff of each unit on the care of the ECMO patient and the use of the bedside reference manual. ECMO patients are the most critical patients at ACH. It is very important to have a formal orientation and reference manual to assist the nursing staff to provide the intensive care needed for positive patient outcomes. After completion of the inservices, nursing staff from the three units demonstrated renewed interest and enthusiasm, and verbally expressed increased comfort while providing nursing care to the patient on ECMO.

\section{Fetal ventricular arrhythmias: diagnosis, prognosis and management}

\author{
P. L. Ferrer, ${ }^{1,2}$ C. Ratnasamy, ${ }^{1}$ A. Parthiban, ${ }^{1}$ J. Osit,${ }^{2}$ M. Young, ${ }^{1}$ G. Wolff, ${ }^{1}$ M. Rodriguez ${ }^{3}$ \\ Departments of ${ }^{1}$ Pediatrics, ${ }^{2}$ Obstetrics and ${ }^{3}$ Pathology, University of Miami School of Medicine, Miami, \\ Florida, United States of America
}

Purpose: The aim of this study was to review our clinical experience with ventricular arrhythmias in the fetus (1983-2004). Methods: Six hundred and eight (608) fetuses with arrhythmias were reviewed; 22 of them with ventricular arrhythmias were selected for presentation. The diagnosis of arrhythmia was made by simultaneous recordings of atria and ventricles, by dual M-mode and Doppler echocardiography. Data obtained was compared with a normal control group of 91 fetuses according to gestational age (GA). Results: Sixteen fetuses had ventricular extrasystoles (VEs), 13 of them not associated with structural heart disease; two had ventricular septal defects and one a complex form of dextrocardia and atrioventricular canal, malposition of the great arteries, and pulmonary atresia. All cases subsided prenatally or postnatally. Fifteen survived; the fate of one is unknown. Six fetuses had ventricular tachycardia (VT) pre or postnatally; all had VEs. Three fetuses had non-sustained VT, two of them accelerated idioventricular rhythm, all subsided postnatally. Two of them had no structural heart disease and one fetus had mild hypertrophic cardiomyopathy. Three fetuses had an unusual course. One fetus diagnosed with perinatal hydrops fetalis and supraventricular tachycardia was converted to normal sinus rhythm shortly after birth. Nonetheless, he developed VT, ventricular fibrillation and died; the autopsy showed oncocytic cardiomyopathy. A second fetus, diagnosed at 32 weeks of GA with multiple rhabdomyomas of both ventricles and atrial extrasystoles, developed incessant VT at 3 months of age, unresponse to antiarrhythmic therapy. This infant underwent a life saving ablation of the site of origin of the VT in the right ventricle. A third fetus followed with the diagnosis of 2 nd degree AV block, was transferred to us in late pregnancy, developed VT, non-sustained ventricular fibrilloflutter and rapid onset of hydrops fetalis requiring emergency 
C-section. Postnatally, it proved to be a case of prolonged QT syndrome. VT was resistant to antiarrhythmic therapy requiring multiple conversions. A left stellate ganglionectomy was unsuccessful; eventually required cardiac transplant. Conclusion: Fetal ventricular arrhythmias are infrequent, 3.6\% of all arrhythmias (2.6\% for ventricular extrasystoles, $1.0 \%$ for ventricular tachycardia). The risk of VEs developing ventricular tachycardia is unknown; in this series it was $27.3 \%$. Ventricular extrasystoles and non-sustained ventricular tachycardia were treated conservatively. Occasionally, management may require emergency intervention, such as early delivery, cardioversion, radiofrequency ablation, antiarrhythmic therapy, and even cardiac transplant. Death is not inevitable; our overall survival was $21 / 22$ patients $(95.5 \%)$.

\title{
Comparison of the utility of CT angiography and magnetic resonance imaging in clinical practice
}

J. D. Reich, G. Bradley, H. Gorrell, M. Poutsch-Amin

The Watson Clinic LLP, Lakeland, Florida, United States of America

Introduction: Although MRI is considered the imaging modality of choice for aortic arch anomalies and nonechocardiographic imaging in complex adult congenital heart disease, few studies have compared either the relative advantages or the clinical results of CT angiography (CTA) and MRI. Study design: Retrospective review of patients in a single pediatric and adult congenital heart disease practice over 4 years referred for either CTA or MRI. Methods: Patients were imaged either in clinic, 4 surrounding emergency rooms, or after referral to tertiary care surgical centers. The Watson Clinic LLP, the primary out-patient clinic location, was without cardiac MRI facilities until 2004. Results of the imaging modality were graded on clinical outcome of the imaging (incorrect or misleading, medically definitive, surgically definitive, or inconclusive). Results: 32 patients were referred for CTA and 10 for MRI. The average age (CTA 20.4 years, MRI 21.0 years) and the indications for the studies were not significantly different. Follow-up or diagnosis of coarctation of the aorta was the most common reason for referral (CTA 41\%, MRI 50\%). CTA produced 2 misleading studies, 19 medically definitive studies, 8 surgically definitive studies, and 1 inconclusive study. Two patients were lost to follow-up. The 8 surgical patients were presented on 9 occasions at 5 different surgical centers. No surgical patient had an MRI requested. MRI produced 1 incorrect study, 1 misleading study, 5 medically definitive studies, and 2 inconclusive studies. One study was aborted when the patient was found to have a residual pacing wire. Conclusion: In this non-randomized study, CT angiography was utilized for roughly the same indications as MRI. CT angiography performed as well as MRI in the diagnosis and referral of congenital heart disease for intervention.

\section{Efficacy of implantable cardioverter defibrillator in children with dilated cardiomyopathy}

\author{
P. Rusconi, D. Lange, G. S. Wolff, E. R. Rosenkranz, M. L. Young \\ University of Miami School of Medicine, Miami, Florida, United States of America
}

Sudden cardiac death (SCD) is a well known risk in patients (pts) with DCMP. This study tests the hypothesis that ICD may prevent SCD in children with DCMP. Methods: The charts were reviewed to determine frequency, type and management of arrhythmias in pts with DCMP referred to our pediatric heart failure clinic from 1/1/98 to 12/31/03. Results: There were 48 pts with DCMP (age range: $0-17.9$ years, mean: $7.1 \pm 6.7$ ) followed up for $3.8 \pm 3.6$ years. All pts were on standard treatment with digoxin, angiotensin converting enzyme inhibitor and diuretics and $75 \%$ were on a beta-blocker. Arrhythmias were detected in $18(38 \%)$ pts: Ventricular tachycardia (VT) in $16(33 \%)$, atrial flutter-fibrillation in $2(6 \%)$. The mean interval from DCMP diagnosis to arrhythmia onset was $3.3 \pm 4.4$ years. There was no significant difference in the left ventricular ejection fraction in pts with or without arrhythmias $(28 \pm 9.8 \%$ versus $24 \pm 5.6 \%, \mathrm{p}=0.14)$. Twelve pts (75\%) with VT had ICD implantation. In a follow-up period of $2.5 \pm 2.0$ years in the ICD pts, there were 26 appropriate shocks ( 0.8 shock per patient-year), and 2 inappropriate shocks ( 0.06 shock per patient-year). Five pts received transplant and 5 pts died in the arrhythmia group (3 SCD; all occurred in pts without ICD, 2 of 
them had no history of VT). In the non-arrhythmia group 12 received transplant and 4 died and none of them had sudden cardiac death. Conclusions: VT is the most common arrhythmia in children with DCMP. Use of ICD can effectively rescue pts from lethal arrhythmias with minimal complications.

\section{Increased left ventricular mass in children after liver and multivisceral transplant}

Y. L. Martinez, O. Gomez-Marin, A. G. Tzakis, O. Garcia, K. Tomoaki, M. Gonzalez II, A. Santiago, P. Rusconi

\section{University of Miami School of Medicine, Miami, Florida, United States of America}

Hypertrophic cardiomyopathy has been reported in patients treated with Tacrolimus after liver and MVT. Objective: to assess the presence of left ventricular hypertrophy (LVH) by quantification of the LVM and to determine possible causes of LVH in the pediatric population that underwent liver or MVT. Method: Medical records were reviewed in the transplanted patients who had at least an echocardiogram. Cross-sectional bivariate and multivariate analyses were performed to assess the relationship between LVM calculated by echocardiogram and blood pressure, tacrolimus level and dose of steroids at the time of the echocardiograms. Result: From 1/1/00 to 4/1/03 39 of 116 children, who underwent liver or MVT at our institution, had echocardiograms available for review. There were 22 females (56\%). Mean age $3.4 \pm 4.7$ years. Mean follow-up $1.7 \pm 0.9$ years $(0.1-3.4$ years). Increase in LV mass above normal was noticed in 10 patients pre-transplant, 13 early and five late after transplant. LV mass returned to normal in 13 patients. Tacrolimus level and the use of steroids were not significant predictors of increased LV mass, but hypertension was an independent predictor at 1 year and 2 years posttransplant. Increased LV mass at pre-transplant was predictive of increased LV mass at 6-12 months post-transplant. Conclusion: The presence of significant increase in the LV mass post liver, or MVT indicates the need to perform pre transplant echocardiograms as baseline and continue follow up of the LV mass to assess for the development of hypertrophy. Better blood pressure control may prevent development of LVH. Prospective studies are necessary to confirm these results.

\section{Combined cryotherapy/RF energy approach to ablation of supraventricular tachycardia in pediatric patients}

J. Philip Saul, Aya Miyazaki, Thomas Kriebel, Claudia Broistedt, Thomas Paul, Andrew D. Blaufox

MUSC, Charleston, Herz Zentrum Gottingen, Gottingen, Germany

Background: Catheter cryoablation (CA) may be useful for multiple forms of supraventricular tachycardias (SVT), including AV nodal reentry tachycardia (AVNRT), AV reciprocating tachycardia (AVRT), junctional ectopic tachycardia (JET), and atrial ectopic tachycardia (AET). However, minimal data on its safety and effectiveness exist in pediatric pts. Methods: Fifty-eight pediatric pts (age: median $=12.8 \mathrm{yrs}, 1-21$; wt: $49 \mathrm{~kg}$, 15-130) underwent CA at two institutions. 25 had AVNRT, 31 AVRT (14 right septal, 9 right free wall, 10 left free wall), 1 AET and 1 JET. Three pts with AVNRT had congenital heart disease (CHD). Applications were cryomaps $(\mathrm{CM})$ if they were $<120 \mathrm{~s}$ or if the min tip temperature was $>-35^{\circ} \mathrm{C}$. Other lesions were considered CAs. Results: $226 \mathrm{CMs}(3 / \mathrm{pt}, 1-24)$ and $74 \mathrm{CAs}(1 / \mathrm{pt}, 1-4)$ were performed, for a total cryo time of $689 \mathrm{~s} / \mathrm{pt}$ (66-2732). Procedural success with CA was achieved in 40 of 57 pts (21/25 AVNRT, 17/30 AVRT, 1/1 JET, 1/1 AET). Total \# of applications (CM + CA) was greater for AVNRT than AVRT (11.8 \pm 9.7 vs $6.0 \pm 4.5, \mathrm{p}<0.05)$, while the number of $\mathrm{CM}$ or CA alone trended higher for AVNRT $(\mathrm{p}=0.08)$. For AVNRT, success was associated with fewer CMs and CAs $(3.5 \pm 7.6$ vs $16.5 \pm 8.3, \mathrm{p}<0.05$, and $2.2 \pm 1.2$ vs $5.0 \pm 2.7, \mathrm{p}<0.01)$, and less total cryo time $(881 \pm 527$ vs $2101 \pm 679 \mathrm{~s}, \mathrm{p}<0.05)$. For AVRT, permanent success occurred earlier after reaching $-25^{\circ} \mathrm{C}$ than transient success $(2.3 \pm 3.5$ vs $23.8 \pm 12.8 \mathrm{~s}$, $\mathrm{p}<0.05)$ and was not achieved after $6 \mathrm{~s}$ : however, the \# of CMs or CAs, total cryo time, and pathway location were not related to success. Success was achieved in $33 \%$ with CHD and $86 \%$ without, $\mathrm{p}=\mathrm{NS}$. Of the 17 pts 
with failed CA, 15 were ablated successfully with RF energy (total success 55/57, 96.5\%). Transient 1 st or 2 nd degree AV block occurred with 7 applications, but there were no permanent complications. Recurrence was seen in 5 pts (2 right septal, 3 AVNRT (2 with CHD)) after 1-40 days. Conclusion: CA is safe for a variety of SVTs in pediatric pts. Success is better for AVNRT than AVRT. Earlier success after tissue freeze may be predictive of a sustained effect. A combined strategy using RF after failed CA can be a safe and effective alternative to RF ablation alone.

\title{
Perioperative hemodynamic effects of nesiritide following cardiac surgery in children
}

\author{
M. Scheurer, ${ }^{1,3}$ J. M. Simsic, ${ }^{1,2}$ J. D. Tobias, ${ }^{1,4}$ J. Berkenbosch, ${ }^{1,4}$ W. S. Schechter, ${ }^{1,5}$ F. Madera, ${ }^{1,6}$
} S. Weinstein, ${ }^{1,7}$ R. E. Michler ${ }^{1,7}$

${ }^{1}$ Heart Care International, ${ }^{2}$ Children's Hospital of Atlanta, Atlanta, Georgia; ${ }^{3}$ Medical University of South Carolina Charleston, South Carolina; ${ }^{4}$ University of Missouri Children's Hospital Columbia, Missouri, ${ }^{5}$ New York,

${ }^{6}$ Santo Domingo, Dominican Republic, ${ }^{7}$ Columbus, United States of America

Background: Nesiritide (BNP; Natrecor, Scios Inc) is a human B-type natriuretic peptide produced by recombinant technology. Nesiritide has hemodynamic effects that may be beneficial in pediatric patients after cardiac surgery. However, limited experience with Nesiritide and pediatrics is reported in the literature. The purpose of this study was to evaluate the safety of Nesiritide in pediatric cardiothoracic surgery. Methods: The study was approved by the Ministry of Health, Dominican Republic. Seventeen patients with congenital heart disease were treated with BNP during cardiac surgery. A loading dose $(1 \mathrm{mcg} / \mathrm{kg})$ was given to each patient while on cardiopulmonary bypass (constant flow) and a continuous infusion maintained for 24 postoperative hours $(0.01 \mathrm{mcg} / \mathrm{kg} / \mathrm{min} \times 6 \mathrm{hrs}$, then $0.02 \mathrm{mcg} / \mathrm{kg} / \mathrm{min} \times 18 \mathrm{hrs})$. The median age was 8 yrs (range $0.3-14 \mathrm{yrs}) ;$ median weight was $21 \mathrm{~kg}$ (range 4-55 kg). Surgical procedures included repair of TOF (9); repair of VSD (7); mitral valve repair (1). Results: The mean cardiopulmonary bypass time was $130 \pm 56$ min; mean cross clamp time was $70 \pm 48 \mathrm{~min}$. Mean blood pressure while on cardiopulmonary bypass decreased by $7 \pm 7 \%$ after BNP load (pre BNP $54 \pm 12 \mathrm{mmHg}$ versus post BNP $50 \pm 12 \mathrm{mmHg}$; p-value 0.003) (14 of 17 patients). In 15 of 17 patients, BNP was the only postoperative medication; 2 were also on milrinone. No patient required additional volume or inotropic support during the BNP load or in the ICU during the first 24 postoperative hours. All patients received their cell saver blood during the first 4 postoperative hours. Lasix $(1 \mathrm{mg} / \mathrm{kg} / \mathrm{dose})$ was started on postoperative day 1 in all patients. Two patients continued BNP infusion past the 24-hour period. None of the patients had arrhythmia. The mean total input was $82 \pm 41 \mathrm{cc} / \mathrm{kg} /$ day; mean urine output was $4 \pm 2 \mathrm{cc} / \mathrm{kg} / \mathrm{hr}$. Conclusions: A $7 \%$ decrease in mean blood pressure was seen following BNP loading dose on cardiopulmonary bypass. No patient required intervention for hypotension while receiving BNP load or infusion. BNP load during, and continuous infusion after cardiac surgery in pediatric patients resulted in no significant hemodynamic compromise.

\section{Reductions in parental separation anxiety and self-reported anxiety in pediatric heart patients who attend a 1-week pediatric camp}

L. E. Simons, ${ }^{1}$ R. M. Campbell, ${ }^{2}$ R. L. Blount ${ }^{1}$

${ }^{1}$ University of Georgia E University of Florida, Florida; ${ }^{2}$ Emory University, Atlanta, Georgia, United States of America

Introduction: The goal of this study was to examine the effects of Camp Braveheart, a summer camp specifically designed for children with complex cardiac abnormalities on a child's emotional adjustment, social functioning, and quality of life. Method: Participants consisted of 29 children who attended Camp Braveheart in July 2003 and completed measures prior to attending camp, on the last evening of camp, and at 2-month follow-up. Data were collected from each child and the child's parents. Results: Repeated measures analyses to assess the impact of camp indicated reports of anxiety decreased from pre-camp $(M=12.0, S D=7.0)$ to at-camp $(M=10.3, S D=6.2)$ on levels of anxiety, $F(1,28)=4.95, \mathrm{p}=.034$. No change was found on measures of 
attitude toward illness, depression, and quality of life (cardiac and general). Parental reports of separation anxiety significantly decreased from pre-camp $(M=62.9, S D=9.7)$ to follow-up $(M=59.1, S D=10.2)$ on parental separation anxiety, $F(1,17)=4.81, \mathrm{p}=0.042$. Parents did not report significant psychosocial changes in their children two months following camp. In addition, reports indicated that children had a positive socialization, normalization, and esteem-enhancing experience at camp. In addition, attitudes toward the camp experience significantly improved at follow-up. Conclusions: The reduction in anxiety observed during the camp experience provides support for the psychological benefits of camp and an incentive for children who have never attended to potentially reap the same positive benefits. The significant decrease in parental separation anxiety is a new, promising finding. A child's ability to succeed independently in the camp environment demonstrates to the parent that the child can move away from overdependence, a dilemma parents of chronically ill children often face. Reports of positive peer relations and feeling normal at camp carry out the goals set for pediatric camps. The improvements in children's attitudes about camp may be due to the persistence of benefits of the camp in the child's life and as memories focus on the overarching themes of camp, such as group support and making friends. 